\section{ambient \\ SCIENCE \\ Vol. 06h(1):40-42 \\ Year 2019}

ORIGINAL ARTICLE

\title{
Saving from Fatal Effect of Aluminum Phosphide (Rice Tablet): a Case Report
}

\author{
Esmail Rayat Dost', Mahdi Foroughian', \\ Masihollah Shakeri ${ }^{3 *}$, Heshmatollah \\ Shakeri ${ }^{4}$, Samaneh Abiri ${ }^{1}$ \\ 'Department of Emergency Medicine, Jahrom University of \\ Medical Sciences, Jahrom, Iran \\ ${ }^{2}$ Department of Emergency Medicine, Mashhad University of \\ Medical Sciences, Mashhad, Iran \\ ${ }^{3}$ Department of Internal Medicine, Jahrom University of Medical \\ Sciences, Jahrom, Iran \\ ${ }^{4}$ Department of infectious disease, Jahrom University of Medical \\ Sciences, Jahrom, Iran. \\ Study Area: Jahrom, Iran \\ Coordinates: $28^{\circ} 30^{\prime} \mathrm{oo} " \mathrm{~N} ; 53^{\circ} 33^{\prime} 38: \mathrm{E}$
}

\section{Abstract}

Aluminum phosphide (rice tablet) compound is a common cause of adult poisoning in Iran. This report deals about successful treatment of one case of poisoning with two rice tablets. An 18-year-old man visited Peymaniye Hospital in complete consciousness but vomited repeatedly and complained about abdominal pain. After going through the prescribed necessary medical aid, the patient was transferred to the intensive care unit (ICU) and was rescued from death. This showed that careful and continuous patient care, immediate transfer of the patient to the ICU, controlling hemodynamic changes, treating acid-base and electrolyte disorders can help to reduce mortality rates caused by severealuminum phosphide poisoning.

Key words: Phosphine gas, Magnesium Sulfate , SorbitolCharcoal, Mineral oils

phosphide poisoning (Chugh et al., 1997; Abbaspour et al., 2013; Khodabandeh et al., 2014). Various studies have reported that mortality rates vary from $50 \%$ to $100 \%$ (Abder-Rahman et al., 2000; Shadnia et al., 2008). Over $1.5 \mathrm{~g}$ dose of the rice tablet is fetal (Singh et al., 1996). There are two types of rice tablet poisoning; if the patients took water-exposed and ineffective rice tablets, they will immediately throw up and have mild aluminum poisoning. The symptoms are headaches, dizziness, nausea, vomiting, and dyspnea. This type of poisoning can be cured with supportive therapies (Rahbar Taramsary et al., 2006). As per the available case reports the mortality rates of aluminum phosphide poisoning vary between $18.6 \%$ and $24 \%$ in Iran. Previous studies have shown high mortality rates of this poisoning in rural areas in Iran, however it was without considering the gender variable (Nosrati et al., 2013). There is no definite antidote for aluminum phosphide poisoning. Therefore, it is important to increase knowledge on suitable and acceptable treatment of this type of poisoning among poison treatment centers personnel to increase survival rates of poisoned patients (Shadnia et al., 2009)..

\section{Case history:}

About the patient: an 18-year-old patient, swallowed two rice tablets admitted to the Emergency Department of the Peymaniye Hospital in Jahrom at $19.30 \mathrm{hr}$, accompanied by 
one of his friend. He was vomitting repeatedly but was alert and aware where he was and what time was it. Initial vital parameters were blood pressure - 95/6o, RR - 100, O2 SAT $97 \%$. He complained about abdominal pain. Intravenous bolus injection of normal saline was immediately administered to the patient followed by the stomach wash thoroughly with diluted permanganate solution and aloe juice. Then, olive oil and charcoal were respectively administered to the patient. Simultaneously, N-acetyl Cysteine (NA), Magnesium Sulfate, Calcium Gluconate and Glucose + Insulin were administered to the patient. The patient suffered from hypotension. Dopamine was administered immediately. He was also injected with $500 c c$ voluven serum and Bicarbonate was administered both intravenously and orally. Further, the patient was transferred to the ICU. He started to complain about his abdominal pain again in the ICU. Thus, Olive oil was administered to the patient at a dose of $150 c c$ every 2 hours. Unfortunately, the patient got diarrhea, therefore, olive oil administration stopped immediately.

The second day in ICU: the doses of Calcium Gluconate and NAC were reduced. Charcoal and Glucose + Insulin were also discontinued. The patient noticed to get suffering from hypotension at $8 \mathrm{pm}(7 \mathrm{o} / 6 \mathrm{o})$. Thus, the dose of Glucose Gluconate was increased. Further, Hydrocortisone was administered and the patient's serum was changed to normal saline. Two hours later, the patient's blood pressure improved and he regained complete consciousness.

The third day in the ICU: the patient seend to be suffered from acidosis ( $\mathrm{pH}=7.18$ and $\left.\mathrm{HCO}_{3}=6.8\right)$, Blood Glucose level was checked (320). An internist diagnosed him with diabetic ketoacidosis (DKA). DKA was treated with Serum therapy + Insulin. DKS subsided completely within 6 hours $\left(\mathrm{PH}=7.38\right.$ and $\left.\mathrm{HCO}_{3}=18.6\right)$. The patient was on Per Oral.

The fourth day in the ICU: the insulin doses were adjusted and the patient was completely in conscious. NAC was discontinued and the dose of Hydrocortisone was reduced.

The fifth day in the ICU: Calcium Gluconate and Hydrocortisone werealso discontinued.

The sixth day in the ICU: The patient was completely recovered and vital symptoms were get normal.

\section{Discussion:}

Proper care of the patient in the initial hours of consuming the aluminum phosphide poisoning is the most important factor for the successful recovery of the patients. The patient care and treatment should be closely monitored from the time of admission to the emergency room to transfer to the ICU (Reza \& Hasan, 2007). Hypotension was noted to be so severe in such patients that continuous infusion of isotonic serum which does not suffice to raise blood pressure. Dopamine should be inevitably administered to raise blood pressure. Administration of continuous infusion of Sodium bicarbonate was seen to be beneficial to subside acidosis (Singh et al., 1980; Schonwald, 2001; Dart, 2004). However, hemodialysis could also be replaced with Sodium Bicarbonate in case of hypervolemia (fluid overload) (Singh et al., 2006). The patient also suffered from severe hypotension in this study. Dopamine was administered immediately. The voluven serum (500 cc) was also administered. The patient was administered bicarbonate both intravenously and orally. New reports have emphasized on administration of Calcium and Magnesium. It is recommended to use Magnesium Sulfate for treatment of Aluminum Phosphide poisoning due to the high prevalence of Hypomagnesaemia in the course of Aluminum Phosphide poisoning and its relationship with fetal arrhythmias and death. Many studies have already reported about the lower rates of mortality in case of treatment with Magnesium Sulfate (Siwach et al., 1994; Chugh et al., 1994; Chugh et al., 1997). It is recommended to use $10 \mathrm{mg}$ Calcium Gluconate $10 \%$ for membrane stabilization. Gluconate or Calcium Chloride is also recommended for the treatment of tetanus (O'Malley et al., 2013; Mehrpour et al., 2011). Magnesium Sulfate is a membrane stabilizer that reduces mortality rates against Aluminum Phosphide poisoning (Chugh et al., 1994a,b). It also has antioxidant properties which inhibit the free radicals released by Phosphine (Chugh et al., 1997; Duenas et al., 1999). Overall, Magnesium Sulfate reduced mortality rates against Aluminum Phosphide poisoning by 50\% (Chugh et al., 1991; Chugh et al., 1994a,b; Gupta et al., 1995). Reza \& Hasan (2007) also used Magnesium Sulfate and Calcium Gluconate to treat the patientsagainst Aluminum Phosphide poisoning.

Repeated gavage of Sorbitol-charcoal and mineral oils help to restrict the release of phosphide and accelerate the excretion of phosphide (Singh et al., 1985; Schonwald, 2001; Dart, 2004). Charcoal and olive oil were also used in this study.

Previous assessments have shown that NAC is suitable for treatment of ALP. NAC can increase the level of Glutathione and Magnesium. It is also an antioxidant (Chugh et al., 1997). NAC also increases survival time and reduces intubation duration and mechanical ventilation (Siwach et al., 1995). It also increases the blood pressure and maintains normal blood pressure within the first 24 hours following ALP. In the present case, NAC was also used to treat ALP.

Conclusively, the Poisoning with Aluminum Phosphide is very common and fatal. The victims suffer from extensive side effects and multi-organ failure and eventually die. Successful treatment of the patient in this study showed that timely drug treatment and continuous carefully patient care including immediate transfer of the patient to the intensive care unit, control of hemodynamic changes, subsiding acidosis and treatment of electrolyte 
disorders can somehow reduce the mortality rates, even in cases of severe poisoning.

Acknowledgment:

We would like to thank the Hospital staff for proper cooperation during the treatment

\section{References:}

Abbaspour, A., Nasrabadi, Z.N., Ghorbani, A. \& Marashi, S.M. (2013): Successful treatment of acute aluminum phosphide poisoning induced heart failure: a case report. $\underline{\text { Razi J. Med. }}$

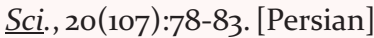

Abder-Rahman, H.A., Battah, A.H., Ibraheem, Y.M., Shomaf, M.S. \& Ei-Batainch, N. (2000): Aluminum phosphide fatalities, new local experience. Med. Sci Law, 40(2):164-168.

Chugh, S., Chugh, K., Arora, V., Kakkar, R. \& Sharma, A. (1997a): Blood catalase levels in acute aluminum phosphide poisoning. L. Assoc. Physicians India. 45(5):379-380.

Chugh, S.N., Kolley, T., Kakkar, R., Chugh, K. \& Sharma A. (1997b): A critical evaluation of anti-peroxidant effect of intravenous magnesium in acute aluminium phosphide poisoning. Magnes Res., 10(3):225-230.

Chugh, S.N., Kumar, P., Aggarwal, H.K, Sharma, A., Mahajan, S.K. \& Malhotra K.C. (1994): Efficacy of magnesium sulphate in aluminium phosphide poisoning--comparison of two different dose schedules. J. Assoc. Physicians India., 42(5): 373-375.

Chugh, S.N., Jaggal, K.L., Sharma, A., Arora, B. \& Malhotra, K.C. (1991): Magnesium levels in acute cardiotoxicity due to aluminum phosphide poisoning. Indian J. Med. Res., 94:437439.

Chugh, S.N., Kamar, P., Sharma, A., Chugh, K., Mittal, A. \& Arora, B. (1994): Magnesium status and parenteral magnesium sulphate therapy in acute aluminum phosphide intoxication. Magnes. Res., 7(3-4):289-294.

Dart, R.C. (2004): Medical toxicology, 3rd ed, Pub. by: Lippincott, Williams and Wikins, Philadelphia, PA. P. 2304.

Dueñas, A., Pérez-Castrillon, J.L. Cobos, M.A. \& Herreros, V. (1999): Treatment of the cardiovascular manifestations of phosphine poisoning with trimetazidine, a new antiischemic drug. Am. J. Emerg. Med.,17(2):219-220. Gupta, S. \& Ahlawat, S.K. (1995): Aluminum phosphide poisoning--a review. I. Toxicol. Clin. Toxicol., 33(1):19-24.
Reza, K. \& Hasan, T.. (2007): A report of two successful treatment cases of rice pill poisoning. J. Babol Univ. Med. Sci., 9 (1):5862.

Khodabandeh, F., Kahani, A. \& Soleimani, G. (2014): The study of fatal complications of "rice tablet "poisoning. Iranian J. Forensic Med., 20(2)27-36.[Persian]

Mehrpour, O., Farzaneh, E. \& Abdollahi, M. (2011): Successful treatment of Aluminum Phosphide poisoning with digoxin: a case report and review of literature. Int. J. Pharmacol., 7(7): 761-764.

Nosrati, A., Karami, M. \& Esmaeilnia, M. (2013): Aluminum phosphide poisoning: a case series in North Iran. Asia Pac. J. Med. Toxicol., 8(3):111-113.

O'Malley, M., Fong, H., Sánchez, M.E., Roisman, R., Nonato, Y. \& Mehler, L. (2013): Inhalation of phosphine gas following a fire associated with fumigation of processed pistachionuts. $I$. Agromed.,18(2):151-173.

Rahbar Taramsary, M., Orangpoor, R., Zarkami, T., Palizkar, M. \& Mousavian, S. (2006): Survey Patients Poisoned With Aluminum Phosphide (Rice Tablet). J. Guilan Univ. Med. Sci., $14(56): 42-47$.

Schonwald, S. (2001): Medical Toxicology, a Synopsis and Study Guide, 1st ed, Pub. by: Lippincott, Williams and Wilkins. P. 768.

Shadnia, S., Mehrpour, O., Abdollahi, M. (2008): Unintentional poisoning by phosphine released from aluminum phosphide. Human Exp. Toxicol., 27(1):87-89.

Shadnia, S., Sasanian, G., Allami, P., Hosseini, A., Ranjbar, A., Amini-Shirazi, N.\& Abdollahi, M. (2009): A retrospective 7years study of aluminum phosphide poisoning in Tehran: opportunities for prevention. Human Exp. Toxicol., 28(4): 209-213. 